\title{
Study on Surgical Re-Admission in DMCH
}

\author{
M M HUSSAIN $^{\mathrm{a}}$, AAM CHOWDHURY ${ }^{\mathrm{b}}$
}

Summary:

Introduction: Re-admission of surgical patients following discharge from a surgical care unit to the same or different hospital is not uncommon. Underlying causes varied. Majority of re-admission are unplanned. Postoperative complications are mainly responsible for patients to seek re-admission. Post operative complications are the mainly liable for surgical re-admission.

Methods: A prospective study was performed in General Surgical units of Dhaka Medical College Hospital, Dhaka for one year from August'2011 to July'2012. Amongst the patients admitted in surgical units, all re-admitted patients within the period had been studied. Purposive sampling was done. Rate, cause, avoidability etc. were studied according to criteria.

\section{Introduction:}

Numerous indicators may be used to evaluate the quality of hospital services, including readmission rate. The readmission rate may reflect the impact of hospital care on the patient's condition upto the point of discharge, as well as describing the efficiency of the service. The readmission rate is easily calculated by hospital information system and may be readily combined with other data ${ }^{1}$.

Hospital readmission can be defined as patient admission to a hospital within a certain period after discharge ${ }^{2}$. The time span varied in literature from thirty days to several years ${ }^{3}$. Some of the used definitions of re-admission are, "the next subsequent admission of a patient as an immediate (that is, emergency or unplanned) admission to any hospital within the same district, within a defined interval of a

\footnotetext{
a. Prof. Dr Md. Margub Hussain, Retired Professor of Surgery, Dhaka Medical College and Hospital, Bangladesh.

b. Dr Abdullah Al Mamun Chowdhur, Medical Officer, Surgery, Upazilla Health Complex, Chandanaish, Chittagong

Address of Correspondence: Dr. Abdullah Al Mamun Chowdhury, Medical Officer, Surgery, , Upazilla Health Complex, Chandanaish, Chittagong, Mobile: 01716566299,E-mail: aamc235@gmail.com

Received: 22 November, 2016
}

Accepted: 15 October, 2018
Results: Among 4396 admitted patients 294 (7\%) had history of previous admission in surgical department of different hospitals. $65 \%$ patients were male and $70.07 \%$ were middle aged (20-50yr). 67\% re-admisssion occurred through emergency department of which $67.7 \%$ were unplanned. $58 \%$ re-admission were avoidable. Diagnosis at readmission contains quite a long list but notables were loop ileostomy (23.47\%), enterocutaneous fistula (10.54\%), incisional hernia (9.52\%), mature colostomy (8.84\%), wound infection (8.5\%) and subacute intestinal obstruction (4.76\%).

Conclusion: Re-admission can be a contributing factor to assess quality and performance. It should be focused in audit and practice.

(J Bangladesh Coll Phys Surg 2019; 37: 54-59)

DOI: https://doi.org/10.3329/jbcps.v37i2.40560

previous (index) discharge taking place within a defined reference period"4. Other views are hospital readmission as an inpatient admission of a patient to a hospital within 90 days of an inpatient discharge from the same hospital ${ }^{5}$. In one literature they defined, hospital readmission can be defined as patient admission to a hospital within a certain period after discharge from the same hospital. The time span varied in the literature from thirty days to several years ${ }^{1}$. Another definition is, a second admission, to any hospital in Washington State, within 7 or 30 days of discharge. Crude 7-day and 30-day readmission rates were compared in rural and urban hospitals ${ }^{6}$.

Readmission rates following surgery are an indirect measure of quality of surgical management. The diagnoses commonly observed during readmission (postoperative infection, digestive disorders, and complications of treatment), however, suggest that readmission generally reflects complications of surgery. Some patients develop complications immediately following surgery and remain hospitalized longer, rather than being discharged and readmitted. This may reflect important quality problems that cannot be identified using readmission as a marker. The use of logistic regression analysis, however, controlled for this potentially confounding variable ${ }^{6}$. 
The literature demonstrates that surgical readmissions are more avoidable than medical readmissions $(4,7,8)$. Furthermore, elective surgery is technically performed under planned, controlled conditions, with more thorough preparation by anaesthetic and surgical specialists. The ward is prepared and stuffed for the patient, who is anticipated to have a predictable and stable recovery ${ }^{6}$.

Hospital record linkage is essential for diagnosing readmission accurately. Now a day, it is very easy to develop a database linkage for a hospital and developing it regularly for future practice. Bangladesh is trying to coup up with those trends of modern technologies and digitalization is on process.

\section{Method:}

The observational, prospective, cross-section study was done from August2012 to July 2013 in general surgical wards of DMCH to study the frequency and pattern of surgical re-admission. All patients re-admitted into surgical wards were studied. Data was collected through direct interview and hospital documents. Results were calculated in broad sheets.

Readmission is defined as next subsequent admission of a patient as an immediate admission with an interval. Index admission is defined as previous hospital admission which may be routine or emergency for surgical purpose. Re-admission can be planned, unplanned, avoidable or unavoidable according to initial procedure as well as cause of subsequent admission needed.

Confidentiality and consent were maintained throughout the study. Ethical Clearance Certificate was issued by Chairman, Ethical Review Committee, Dhaka Medical College, Dhaka for the study on 25.02.2012.

\section{Results:}

Within the period, total 4396 patients was admitted to Surgical wards of DMCH from which 294(7\%) patient were selected for the study. $68 \%$ re-admitted patients were male and rest were (32\%) female. among the re-admitted patients $84(28.57 \%)$ were 20-30year age group, $66(22.45 \%)$ were $30-40 y e a r$ age group, $56(19.05 \%)$ were $40-50$ year age group. $65 \%$ of them came from outside the Dhaka and 35\% were from within Dhaka.

$67 \%$ patients were admitted through emergency room and $33 \%$ through elective admission where $67.7 \%$ re- admission were unplanned and $32.3 \%$ were planned (Table I).

Table-I

Types of re-admission

\begin{tabular}{lccc} 
& Planned & Unplanned & Total \\
\hline Emergency & 57 & 139 & $196(67 \%)$ \\
Elective & 38 & 60 & $98(33 \%)$ \\
\hline Total & $95(32.3 \%)$ & $199(67.7 \%)$ & $294(100 \%)$ \\
\hline
\end{tabular}

Table-II

Diagnosis at Re-admission

\begin{tabular}{lc} 
Diagnosis & No. of Patient \\
\hline Mature loop ileostomy & $69(23.47 \%)$ \\
Intestinal leakage (Enterocutaneous fistula) & $31(10.54 \%)$ \\
Incisional hernia & $28(9.52 \%)$ \\
Mature colostomy & $26(8.84 \%)$ \\
Wound infection & $25(8.5 \%)$ \\
Subacute intestinal obstruction & $14(4.76 \%)$ \\
P/O acute abdomen & $12(4.08 \%)$ \\
Post cholecystectomy complication & $11(3.74 \%)$ \\
(pain +/-jaundice) & \\
Abdominal wound dehiscence & $8(2.72 \%)$ \\
Recurrent Ca-Breast & $3(1.02 \%)$ \\
Prolapsed ileostomy stoma & $3(1.02 \%)$ \\
Port site infection & $3(1.02 \%)$ \\
Discharging sinus (on previous scar) & $3(1.02 \%)$ \\
Recurrent inguinal hernia & $3(1.02 \%)$ \\
Reccurent fistula in ano & $3(1.02 \%)$ \\
Billiary peritonitis & $3(1.02 \%)$ \\
Miscellaneous * & $46(15.65 \%)$ \\
\hline Total & $294(100 \%)$ \\
\hline
\end{tabular}

*Post appendectomy septicemia with shock, Billiary fistula with wound infection, Post LUCS acute abdomen, Post operative shock, Amputation stump infection, Recurrent $\mathrm{Ca}$-Colon, Bleeding from stoma site, Recurrence of malignant GIST ( 2 patient each)

P/o scrotal hematoma, Prolapsed colostomy stoma, 9th POD of hernioplasty (Rt sided) with Hemophilia B, Severe anemia with infected amputated stump, Post Gastrojejunostomy electrolyte imbalance, CBD injury, Hepaticolithiasis with Choledocholithiasis, Umbillical port hernia, Reccurent malignant mesenchymal tumor, Post circumcision bleeding, Recurrent abdominal lump (MFH), P/O lymphoedema of lower limb, Retrograde jejunogastric intussusception, Per rectal bleeding, Hypocalcaemic tetany, Bleeding from feeding enterostomy site, Hematuria with Renal failure, Biliary fistula, Hematuria, Recurrent Ca-Stomach, Stromal ulcer, ARF, Malignant GIST in biopsy of appendix, Post tubectomy acute abdomen and shock, Incisional hernia with ITP, Excoriation around stoma, Transection of CBD, Stomal complication with fistula, Urinary fistula, Post splenectomy pyrexia (1 patient each) 
Table-III

\section{Diagnosis in index admission}

Diagnosis at index admission No. of patient

Gastrointestinal

Acute Intestinal Obstruction

Ileal perforation

Ca Stomach

Ileocaecal TB with obstruction 4

Intestinal lymphoma

Colorectal

Acute appendicitis

Sigmoid volvulus

Ca Rectum

Ca-Rectum with obstruction

Ca-Colon

Trauma

Penetrating Abdominal trauma

RTA with polytruma

Hepatobiliary

Choledocholithiasis

Breast \& Endocrine

Ca Breast

Gynaecological

Fibroid uterus

Obstructed labour

Term pregnancy with PROM

Bulky uterus

Miscellaneous*

*Malignant intestinal GIST, ITP (for splenectomy), Fistula in ano, PVD (Buerger's disease?), Term pregnancy with oligohydramnion, Post term pregnancy ( 2 patient each)

Ca Oesophagus, Jejunal adenocarcinoma, Abdominal lump (no description was found), Appendicular abcess, Perianal abcess, Rectal prolapse, Strangulated thrombosed piles, Assault (cut injury over left thigh), Penetrating perineal injury, Accidental rectal injury, Gunshot injury, Barotrauma to rectum, Cholelithiasis, Goiter, Renal cell carcinoma, BEP, Ca-Prostate, Hydrocele (Rt), Varicocele (Rt), Ritual (for circumcision) with Hemophilia A, Rt inguinal hernia with Hemophilia B, Left sided reducible indirect inguinal hernia, Incisional hernia, Umbillical hernia, Malignant mesenchymal sheath tumour, Ruptured ectopic pregnancy, Ovarian cyst, Post DNC complication, Inguinal swelling, Family planning (1 patient each)

\section{Table-IV}

\section{Operation done (during index admission)}

Operation done

No of Patient

Repair of ileal perforation with ileostomy

42

Appendectomy

32

Sigmoid colostomy

26

Cholecystectomy (open)

21

Loop ileostomy

16

Resection and anastomosis of ileum

13

Resection of gangrenous portion with ileostomy

12

LUCS

12

Repair of duodenal perforation

11

TAH

9

Repair of ileal perforation

8

Resection \& anastomosis of ileum with ileostomy

7

Cholecystectomy (lapchole)

Exploratory laparotomy

Hernioplasty

Resection of gangrenous portion with colostomy

Modified radical mastectomy (with

axillary clearance)

Partial gastrectomy with gastrojejunostomy 4

Choledocholithotomy 4

Rt Hemicolectomy 3

Miscellaneous*

46

*Left midthigh amputation, Repair of duodenal perforation with gastrojejunostomy, Appendectomy with loop ileostomy, Feeding enterostomy, Excision of tumour, Excision of tumour, I \& D, Herniorraphy (2 patient each)

Excision \& eversion of sac, Primary repair of wound, Lumpectomy (of breast), Excision of varicocele, Anterior resection with ileostomy, Resection \& anastomosis of sigmoid volvulus, Fistulectomy, Circumcission, Laparotomy \& resection of lump (no description found), Excision of inguinal swelling (left), Hartman's procedure, Resection \& anastomosis, Mucosal resection of rectal prolapse, Thyroidectomy, Ovarian cystectomy, Left sided nephrectomy, TURP, Amputation of 3rd toe (left), Appendectomy \& excision of mesenteric mass, Excision of tumour \& duodenojejunostomy, Prostatectomy, APER, BLTL, Splenectomy, Wound toileting \& suturing, Resection, anastomosis of ileum \& colostomy (?), Fistulectomy (1 patient each) 


\section{Table-V}

Diagnosis in index admission and why re-admission needed

\begin{tabular}{|c|c|c|c|c|c|c|}
\hline \multirow[t]{2}{*}{ Diagnosis } & \multirow{2}{*}{ No. of patient } & \multicolumn{5}{|c|}{ Re-admitted due to (majority cases) } \\
\hline & & $\begin{array}{l}\text { Stoma } \\
\text { closure }\end{array}$ & $\begin{array}{c}\text { Intestinal } \\
\text { leakage }\end{array}$ & $\begin{array}{l}\text { Wound } \\
\text { infection }\end{array}$ & $\begin{array}{c}\text { Abdominal } \\
\text { pain/ acute } \\
\text { abdomen/ } \\
\text { peritonitis }\end{array}$ & $\begin{array}{c}\text { Incisional } \\
\text { hernia }\end{array}$ \\
\hline Acute intestinal obstruction & 25 & 4 & 2 & 7 & 9 & 3 \\
\hline Ileal perforation & 43 & 23 & 7 & 4 & 2 & 2 \\
\hline Acute appendicitis & 35 & - & 5 & 17 & 6 & \\
\hline Sigmoid volvulus & 11 & 7 & - & 3 & & 1 \\
\hline Penetrating abdominal trauma & 15 & 8 & - & 4 & - & 2 \\
\hline RTA with polytrauma & 17 & 6 & - & 7 & - & 3 \\
\hline Choledocholithiasis & 4 & - & - & 1 & 3 & - \\
\hline
\end{tabular}

$37.07 \%$ patients were re-admitted within 1-7days interval, 32.31\% patient within 91-180days, 14.28\% patient re-admitted after one year. The chart also shows that $49 \%$ re-admission occurred within 90 days after discharge. (Fig 1)

$58 \%$ re-admission were avoidable, 34\% were unavoidable (Fig 2)
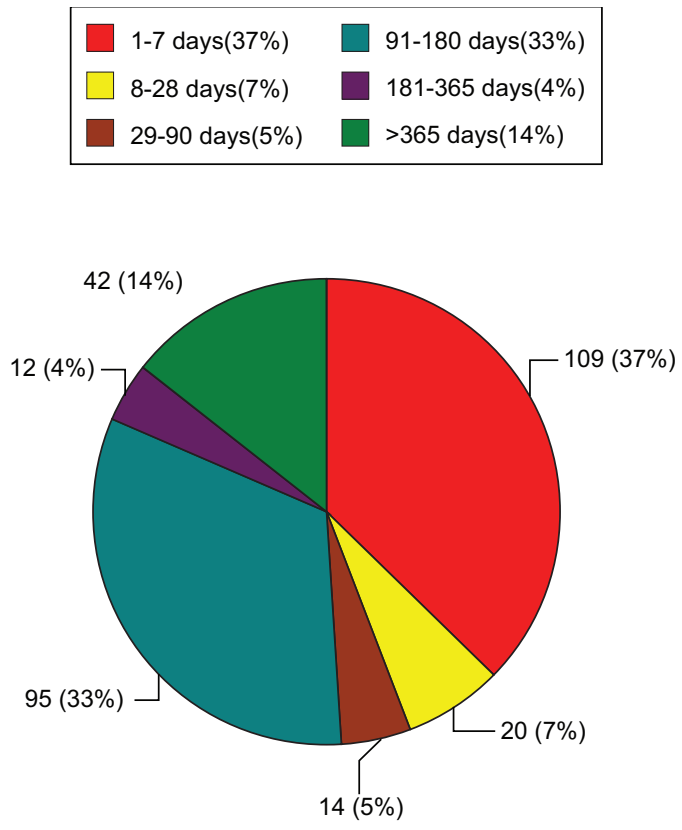

Fig. 1: Interval between two admission

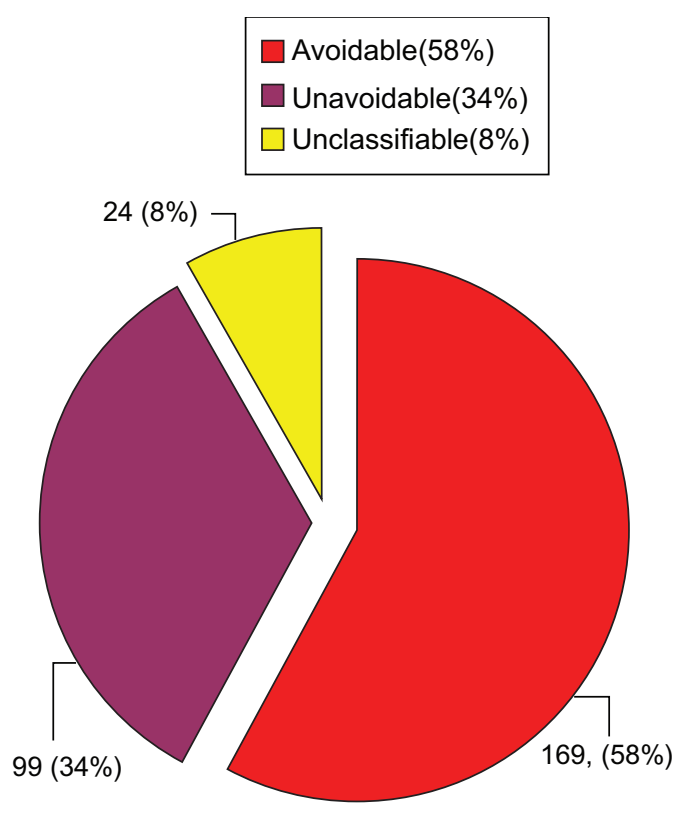

Fig.-2: Type of Re-admission

\section{Discussion:}

Decreasing the incidence of readmission has increasingly become a goal of care givers, hospital administrators, and policy makers ${ }^{9}$.

In this study we found surgical re-admission rate is $7 \%$ (Fig 3.1)which is quite higher than study findings by Hull (4\%), North East Thames ${ }^{7}$ (4.1\%), 
Leicestershire $^{10}$ (4.5\%), and Oxford ${ }^{11}$ (5.3\%). On the other hand, higher readmission rates were recorded for some states of US, such as New Jersey (21.9\%), Louisiana (21.9\%), and Illinois (21.7\%), and for other states, such as Oregon (15.7\%), Utah (14.2\%), and Idaho $(13.3 \%)^{12}$. Surgical re-admission rate in Aga Khan University Hospital, Pakistan was $6.4 \%$ on $2014^{13}$ which was a bit lower than us. But the pitfall is different study targeted different population as some study showed re-admission in total hospital, some showed re-admission for some specific cases. That's why it is difficult to comment about our re-admission rate in context of other studies.

Different co-existing disease plays the vital role and social factor more or less influences the picture. Countries where home delivery of care is lower elderly people commonly brought to hospital. In our study, Dhaka Medical College is the best service provider institute in the whole country. So, some onethird people came here from different districts also.

About $67 \%$ of the re-admitted patients were admitted through emergency department (Table-1) and 67.7\% of the re-admissions were unplanned (Table-1) also. Planned re-admissions in surgical units were mostly for second stage surgery (i.e. stoma closure etc.) or mitigating complications as per advice. Emergency readmissions were commonly for complications (i.e. wound complications, fistula development, acute abdominal conditions etc) or unrelated emergencies. Unplanned emergency re-admission was $47.27 \%$ which indicates mainly surgical complications and can be avoidable to some extent.

Most of the re-admission in DMCH were initially admitted and treated in same institute (i.e. $49.66 \%$ ). It signifies that half of our patients had index admission in $\mathrm{DMCH}$ and admitted here again for planned or unplanned (complicated) indications. This bulk of patient is potentially reducible through regular audit and skilled surgical care.

Surgical re-admission in our study shows a bimodal distribution of patient in Fig-1. 37\% of all patients readmitted within one week of discharge and other 33\% of them admitted again after three to six months of discharge from the hospital. If we compare the data with table-1 and look again the study sheets - we can find that the first group signifies surgical complications and second group surgical plan for re-operation or second stage surgery.

“Are re-admissions avoidable?" was asked by Clarke on 1990. The answer was ambiguous and to some extent it is always true. Especially, surgical enthusiasm and calculating risk-benefit in every step plays an important role in answering avoidability of re-admission in surgery. Avoidibility was between $18 \%$ to $77 \%$ in different studies performed previously ${ }^{10}$. We found in our study that $58 \%$ of all re-admission were avoidable (Fig 2) - if tried.

Hospital stay in index admission affects re-admission rate. Commonly patient discharged earlier without fulfilling discharge criteria shows more incident of re-admission. But in our study we could not find any specific pattern to relate the finding with any other study. A significant portion (i.e. 13.6\%) had hospital stay more than two weeks but admitted again. Most of them were trauma patients needed multiple procedures with stoma.

It is notable that total planned re-admission $(32.3 \%$ in Table 1) were admitted with only two diagnosis i.e. mature ileostomy and colostomy. Other all diagnosis was emergency re-admission. The list of unplanned readmission almost fully deals with operative complications and pitfalls. If proper caution and skill could be applied - many re-admissions can be avoided or at least managed with post operative OPD follow up clinics. We could not judge the conditions about recurrent tumors (i.e. breast, colon, rectum, GIST etc.) as documents contains little about clearance, margin, histopathology, chemo/radiotherapy and follow up.

Complications are more or less part of surgery. No surgery is without some chance of complication. But we urgue that those complications can be avoided if surgeon makes effort and continuous audit and development is needed. Some of those patients may not need any further operation. Sometimes social factors act as avoidable factor i.e. compliance ${ }^{4}$. In our study we described that $58 \%$ re-admission reveals avoidable. Although the proportion of preventable readmissions is variable in the literature, it is agreed that the readmission rate includes a significant fraction of events of ill-health that could have possibly been avoided $^{14-15}$. Our study shows correspondence with the observation of Clarke ${ }^{4}$ as readmissions within week after discharge are significantly more avoidable. 
In 1965, Acheson and Barr suggested that the readmission rate might serve "as an index of the quality of medical care" 16 . This idea has lately regained ground and readmission rates have been described as "one of the few potential measures available from routine statistics for assessing outcome"11. The association between readmission rate and quality of care has been validated in methodologically-sound meta-analyses, cohort and case-control studies ${ }^{17}$. Ideally, outcome indicators should be chosen that measure genuine service objectives ${ }^{4}$. That's why re-admission rate should act as a contributing indicator for quality of care of an institution ${ }^{18}$. Many readmissions represent a failure of the best care ${ }^{19}$. Unplanned readmission might usefully form the focus of practicing audit ${ }^{20}$.

\section{Conclusion:}

The way of surgical improvement is to mitigate the volume of complication and hence re-admission and re-operation also. Our situation is not worse than any standard practice. Still, it demands more caution, more study and more improvement. Best practice of audit can improve our universal performance and patient care.

\section{References:}

1. Castro MSM, Carvalho MS, Travassos C. Factors associated with readmission to a general hospital in Brazil. Cad. Saúde Pública, Rio de Janeiro, 21(4):1186-1200, jul-ago, 2005

2. Evans RL, Hendricks RD, Lawrence KV, Bishop DS, Identifying factors associated with health care use: a hospital based riskscreening index. Soc Sci Med 1988;9:947-53

3. Holloway JJ, Medendoro SV, Bromberg J. Risk factors for early readmission for veterans. Health Serv Res 1990;25:15-37

4. Clarke, A. Measuring readmission rates. BMJ. 1990;301:1134-6

5. GUO JJ, Ludke RL, Cluxton RJ, Moomaw CJ. Characteristics and Risk Factors for Hospital Readmission in the Ohio Medicaid Population. MEDTAPP 2001-2003 Research Project \#2. University of Cincinnati Medical Center College of Pharmacy \& Institute for health policy and Health Service Research Cincinnati, OH 45267, February 12,2003;
6. Welch, H. G., Larson, E. H., Hart, L. G., et al. Readmission after surgery in Washington State rural hospitals. Am J Public Health. 1992;82:407-11

7. Courtney E, Ankrett S, McCollum P. 28-Day emergency surgical re-admission rates as a clinical indicator of performance. Ann $R$ Coll Surg Engl. 2003;85:75-8

8. Anderson G, Steinberg E. Hospital readmissions in the medicare population. NEngl J Med. 1984;311:1349-53

9. Hansen LO, Young RS, Hinami K, et al. Interventions to reduce 30-day rehospitalization: a systematic review. Ann Intern Med 2011;155:520-528.

10. Langley J, Jones J. Use of medical record linkage to study readmission rates [letter; comment]. BMJ 1989; 299: 1032.

11. Henderson J, Goldacre MJ, Graveney MJ, Simmons HM. Use of medical record linkage to study re-admission rates. BMJ 1989; 299: 709-13.

12. Stephen F. Jencks, M.D., Mark V. Williams, M.D., and Eric A. Coleman, M.D., M.P.H., "Rehospitalizations among Patients in the Medicare Fee-for-Service Program," N Engl J Med.2009; 360: 1418-1428.

13. Sultan, R, Abdullah, U, Chawla, T. 30-day readmission rate and its causes in general surgical patients. Journal of the College of Physicians and Surgeons-Pakistan. 2018; Vol. 28 (4): 314-316

14. Maurer P, Ballmer P. Hospital readmissions - are they predictable and avoidable? Swiss Med Wkly. 2004;134:606-11.

15. Miles T, Lowe J. Are unplanned readmissions to hospital really preventable? Jour Qual Clin Prac. 1999;19:211-14.

16. Acheson ED, Barr A. Multiple spells of in-patient treatment in a calendar year. Brit Jour Prev Soc Med. 1965;19:182-91.

17. Ashton C, Del Junco D, Souchek J, et al. The association between the quality of inpatient care and early readmission: a metaanalysis of the evidence. Medical Care. 1997; 35:1044-59.

18. Smith JR, Hider P, Graham P. The readmission rate as an indicator of the quality of elective surgical inpatient care for the elderly in New Zealand. Journal of the New Zealand medical Association, 2009; vol 129 no 1289.

19. Department of Health. Discharge of patients from hospital. London: DOH, 1989. (HC(89)5.)

20. Woodhouse LW. Called to account: a geriatrician. Lancet 1989;i: 1546. 